\title{
FLASHMOB COMO ESTRATEGIA DE MARKETING EN TABASCO, MÉXICO
}

\section{FLASHMOB AS A MARKETING STRATEGY IN TABASCO, MEXICO}

\author{
Analy Hernández Torres*, Manuela Camacho Gómez**
}

* Licenciada en Administración. Universidad Juárez Autónoma de Tabasco, México.

** Doctora en Educación Internacional. Profesora-Investigadora de la División Académica de Ciencias Económico Administrativas. Universidad Juárez Autónoma de Tabasco, México. manuelacamacho@gmail.com

Dirección para recibir correspondencia: analy020293@hotmail.com 


\section{RESUMEN}

OBJETIVO: Analizar el estado del arte del flashmob como estrategia del marketing, sus características, tipología y categorización. La de tipo corporativa ha sido aplicada en España, Asia, Norteamérica y México. En este contexto, se revisan las primeras aproximaciones de organizaciones del Estado de Tabasco que han utilizado esta estrategia.

MATERIAL Y MÉTODO: Este documento es el resultado de una investigación cualitativa con un enfoque inductivo, desarrollada con las técnicas de literaria y análisis de contenido.

RESULTADOS: Las tendencias en el uso del flashmob han sido crecientes. Se trata de una estrategia creativa e innovadora; una herramienta joven del marketing. Su mayor uso se localiza en Europa, Estados Unidos de América y Asia. En el caso de México, se utiliza la categoría de flashmob corporativo. En Villahermosa, Tabasco, México; se localizaron tres organizaciones que lo utilizaron con diferentes propósitos en los años 2012, 2013 y 2015, respectivamente.

CONCLUSIONES: El flashmob es una técnica única y original del marketing. Se desarrolla tomando en cuenta su propósito y la percepción esperada en los segmentos o público objetivo. Existen a la fecha, siete categorías: personal, educacional, actuación artística, política, caridad, criminal y la de negocios o corporativa. En México y en Tabasco empieza a utilizarse principalmente en la categoría corporativa, de caridad y educacional, por lo que sus potencialidades muestran una aplicabilidad efectiva de esta estrategia para posicionar productos y servicios.

PALABRAS CLAVE: Flashmob. Street marketing. Estrategia de marketing. 


\section{ABSTRACT}

OBJECTIVE: Analyze the state of the art of flashmob as a marketing strategy, its characteristics, typology and categorization. The corporate type has been applied in Spain, Asia, North America and Mexico. In this context, the first approximations of organizations from the State of Tabasco that have used this strategy are reviewed.

MATERIAL AND METHOD: This document is the result of a qualitative research with an inductive approach, developed with the literary and content analysis techniques

RESULTS: Trends in the use of flashmob have been growing. It is a creative and innovative strategy; a young marketing tool. Its greatest use is in Europe, the United States of America and Asia. In the case of Mexico, the category of corporate flashmob is used. In Villahermosa, Tabasco, Mexico; Three organizations were located that used it for different purposes in the years 2012, 2013 and 2015, respectively.

CONCLUSIONS: The flashmob is a unique and original marketing technique. It is developed taking into account its purpose and the expected perception in the segments or target audience. Up to now, there are seven categories: personal, educational, artistic performance, political, charitable, criminal and business or corporate. In Mexico and Tabasco, it begins to be used mainly in the corporate, charitable and educational categories, so its potential shows an effective applicability of this strategy to position products and services

KEYWORDS: Flashmob. Streetmarketing. Markting strategies. 


\section{INTRODUCCIÓN}

El marketing es una pieza fundamental en las organizaciones. Sus componentes básicos: producto, precio, promoción y plaza siguen siendo vigentes; sin embargo, nuevas estrategias y formas de lograr los objetivos comerciales o institucionales han cobrado fuerzas a partir del impulso de las tecnologías de información y comunicación. Con estas circunstancias, el ambiente global ha provocado rápidos cambios empresariales y mercados más competidos, lo que ha llevado a las organizaciones a buscar y crear innovadoras estrategias de marketing para atraer a los públicos objetivo, ávidos de: emoción, diversión y nuevas experiencias. El consumidor quiere distraerse, sentir, llenar su vida de nuevas experiencias y disfrutar de su tiempo libre (Catalá y Díaz, 2013).

Es justo en este contexto donde el flashmob tiene cabida, ya que se trata de una estrategia original, creativa y entretenida, tiene como característica principal a la sorpresa que causa entre los asistentes, los cuales se esperaría fueran parte del segmento de interés y entonces, llamar su atención y generarles experiencia agradable y positiva hacia una marca, empresa o institución. Se trata de una acción organizada en la que en un grupo de personas se reúne repentinamente en un lugar público y lleva a cabo un acto inusual que llama la atención y después desaparece, se apoya con aplicaciones móviles, internet y con el boca en boca (Estrella y Segovia, 2016).

El propósito de este artículo es hacer una revisión básica del estado del arte del uso del flashmob y su utilización en empresas de México, particularmente en Tabasco.

\section{Marco Teórico}

El flashmob es parte de las estrategias del denominado Marketing Promocional definido como un grupo de acciones que se caracterizan por incluir estímulos adicionales al producto con el propósito de activar las ventas o la respuesta esperada. La American Marketing Association (AMA), citado por Estrella y Segovia (2016, p. 65), la define como un conjunto de actividades que estimulan las compras de los consumidores y la eficacia de los vendedores, tales como exhibiciones, shows y demostraciones, así como otros diversos esfuerzos de venta no repetitivos que se encuentran fueran de lo cotidiano. Las emociones y el pensamiento lógico, están relacionados con el marketing de contenidos. De acuerdo con Jiménez (s.f., p. 20) es la creación y distribución de contenido relevante para los consumidores y potenciales clientes con el objetivo de atraerlos hacia la empresa y entablar relación con ellos. Este se desarrolla dentro 
FLASHMOB COMO ESTRATEGIA DE MARKETING EN TABASCO, MÉXICO

del marketing digital. Según Andrade (2016), se basa en tres pilares fundamentales: SEO (Search Engine Optimización), marketing de contenidos y social media marketing. Se puede asumir que este tipo marketing proporciona información valiosa, que instruye, divierte, crea emociones y contenidos de calidad que hagan que sea el usuario quien decida lo que desea sobre una empresa, un producto o una marca.

Otra especialidad relacionada con el flashmob es el Street Marketing o marketing de calle, que consiste en llevar a cabo acciones, bien publicitarias, o bien de comunicación, que se efectúan en la calle 0 en entornos exteriores mediante técnicas no controladas por medios convencionales con el fin de generar impactos positivos en los consumidores López (2015, p. 14). Se desarrollan acciones sorprendentes y llamativas que capten la atención del peatón para establecer una relación más inmediata entre el producto y la marca y el cliente potencial (Estrella y Segovia, 2016). Mifflin (s/f, citado por López, 2015), concibe al Street Marketing la consecución de los objetivos del marketing planteados a través de métodos poco convencionales, invirtiendo más que dinero, energía, pasión y creatividad, particularmente con acciones originales e innovadoras. Este incluye al flashmob, una forma de hacer publicidad en el medio urbano con la finalidad de generar un mayor impacto y lograr un acercamiento directo con los consumidores (López, 2015).

Para la organización y desarrollo de un flashmob se requiere un proceso que incluye nueve tareas fundamentales, primeramente definiendo un objetivo claro de lo que se quiere lograr, la gente que va a participar, la logística del evento, la grabación y la posterior difusión en redes y medios sociales (ver figura 1 ).

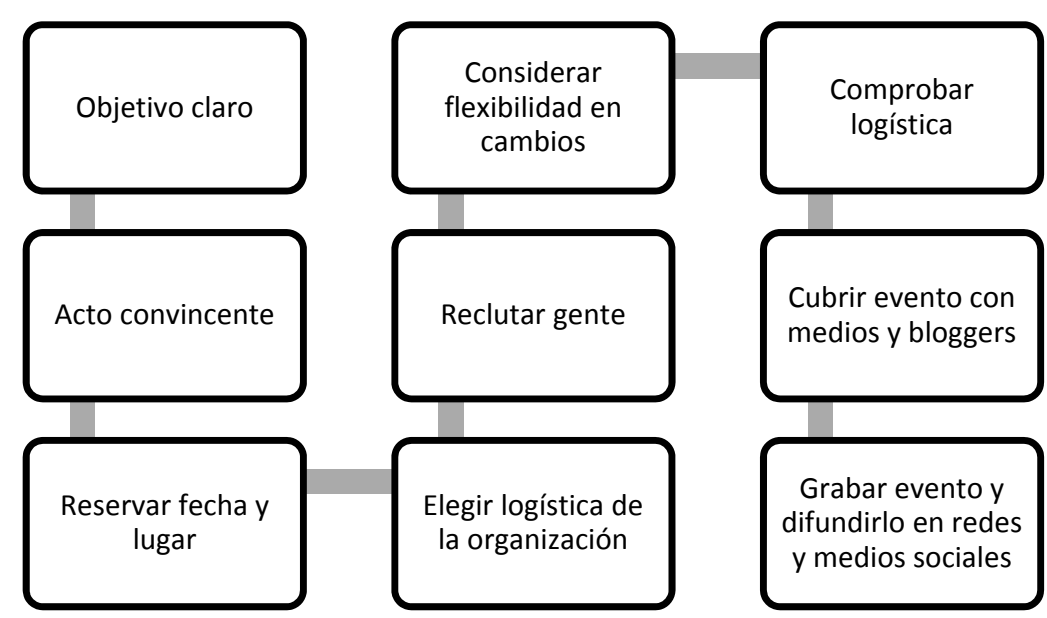

Figura 1. Proceso para el desarrollo de un Flashmob.

Fuente: Melin (2012) citado por Costa (2014, p. 78-79). 
Fraile (2012, p. 334) señala que el flashmob consiste en juntar a un número indeterminado de personas en un lugar público, de manera figuradamente espontánea, para realizar una acción participativa previamente establecida e inusitada para ese contexto, lo más habitual son las coreografías grupales; tras el periodo de realización, normalmente sólo unos minutos, la muchedumbre se disuelve. Pérez y Fanjul (2013) lo traducen como "multitud instantánea". La eficacia reside en su posterior difusión por parte de los propios receptores a través de Internet en canales como YouTube. En términos corporativos el flashmob es una herramienta de comunicación externa que sirve para crear un evento positivo, en un espacio público, en el que participan un grupo de personas (agentes) y espectadores (público presente) y que sirve para sorprender, llamar la atención y crear una experiencia que genere emociones positivas acerca de la marca o de la organización (Costa, 2014, p. 77). Su grabación en video y posterior difusión por medio de la social media garantiza una experiencia de recuerdo que continúa fomentando un entorno emocional positivo.

\section{Categorías del Flashmob}

Respecto a la categorización de los flashmob, Wang, Akella y Bennett (2014), señalan que existen siete propósitos para sus realización: 1) personal, 2) educacional, 3) caridad, 4) actuación artística, 5) negocios, 6) política, y 7) criminal.

\section{Tipología de Flashmob}

De acuerdo a la Universidad de Málaga (España), el flashmob puede clasificarse en siete tipos (ver figura 2).

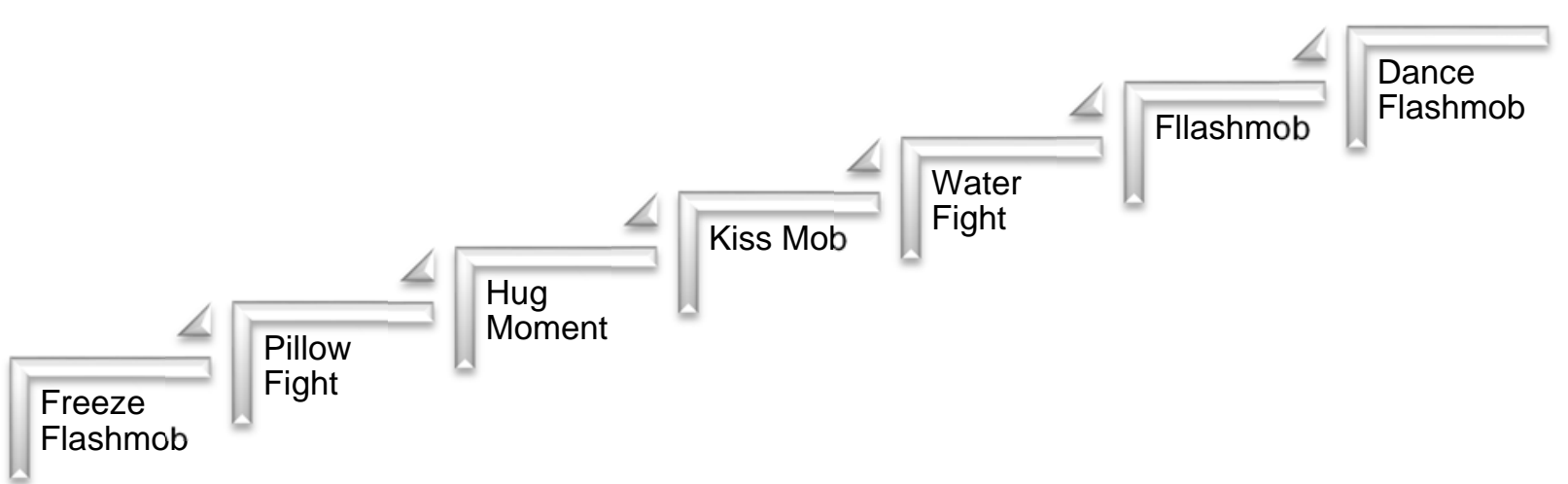

Figura 2. Tipología del Flashmob.

Fuente: Elaborado con datos de www.periodismo.uma.es 
FLASHMOB COMO ESTRATEGIA DE MARKETING EN TABASCO, MÉXICO

Cada tipo de flashmob posee singularidades, debido a que persiguen un propósito en la percepción de sus segmentos o el público al que va dirigido (ver tabla 1).

Tabla 1

Características de diferentes tipos de flashmob

\begin{tabular}{|c|c|c|c|}
\hline Tipo & Característica & Tipo & Característica \\
\hline Freeze Flashmob & $\begin{array}{l}\text { Todos los participantes se } \\
\text { inmovilizan durante varios } \\
\text { minutos como si fueran } \\
\text { estatuas. }\end{array}$ & Pillow Fight & $\begin{array}{l}\text { Al escuchar el sonido de } \\
\text { una trompeta empieza una } \\
\text { guerra de almohadas entre } \\
\text { los participantes. }\end{array}$ \\
\hline Hug moment & Abrazos libres. & Kiss mob & $\begin{array}{l}\text { A la señal todos se besan y } \\
\text { quedan así durante algunos } \\
\text { minutos }\end{array}$ \\
\hline Water Fight & $\begin{array}{l}\text { Suele llevarse a cabo en } \\
\text { lugares al aire libre, ya que } \\
\text { en el momento preciso los } \\
\text { participantes comienzan } \\
\text { una lucha con balones de } \\
\text { agua }\end{array}$ & Dance Flash Mob & $\begin{array}{l}\text { Es el más popular de todos. } \\
\text { En un determinado } \\
\text { momento empieza una } \\
\text { música y todos bailan la } \\
\text { misma coreografía, } \\
\text { previamente acordada } \\
\text { entre los participantes. }\end{array}$ \\
\hline Flash Mob & & \multicolumn{2}{|c|}{$\begin{array}{l}\text { Todos los participantes hacen una misma acción } \\
\text { situados en una fila. }\end{array}$} \\
\hline
\end{tabular}

Fuente: Elaborado con datos de www.periodismo.uma.es

De acuerdo con la literatura revisada, se pudieron localizar datos sobre la tendencia del uso del flashmob en diversas partes del mundo, particularmente a partir de los hallazgos de Costa (2014) y Wang, Akella y Bennett (2014). Según los datos publicados por estos autores, en el periodo del 24 de febrero al 20 de marzo de 2014 se llevaron a cabo un total de 18 actividades de flashmob en España (Costa, 2014); en tanto que en la regiones de Asia y América del Norte, estudiadas en el periodo de 2009 a 2011 y de 2008 a 2011, respectivamente; encontraron un total 100 para Asia y de 97 para América del Norte (Wang, Akella y Bennett, 2014) (ver tabla 2). 
FLASHMOB COMO ESTRATEGIA DE MARKETING EN TABASCO, MÉXICO

Tabla 2

Flashmob en Europa, Asia y América del Norte

\begin{tabular}{|c|c|c|c|}
\hline Continente & Países & Número de flasbmob & Categorías \\
\hline Europa & España & 18 & Negocios \\
\hline Asia & $\begin{array}{l}\text { China, Turquía, Filipinas, } \\
\text { India y Japón }\end{array}$ & 100 & $\begin{array}{l}\text { Personal, educacional, caridad, } \\
\text { actuación artística, negocios, } \\
\text { política, criminal. }\end{array}$ \\
\hline Norteamérica & $\begin{array}{l}\text { Cuba, Canadá, República } \\
\text { Dominicana, México y } \\
\text { Estados Unidos de } \\
\text { América }\end{array}$ & 97 & $\begin{array}{l}\text { Actuación artística, criminal, } \\
\text { personal, negocios, política, } \\
\text { educacional y caridad. }\end{array}$ \\
\hline
\end{tabular}

Nota: Las categorías fueron colocadas en orden de mayor utilización.

Fuente: Costa (2014) y Wang, Akella y Bennett (2014).

La identificación de los flashmob realizados en España en el periodo febrero-marzo, 2014; fueron a través de los canales de YouTube de 100 empresas que pertenecen al índice Monitor Empresarial de Reputación Corportiva (MERCO). El objetivo fue conocer el grado de estandarización del empleo de esta estrategia por parte de las acciones emprendidas por el área de comunicación de estas organizaciones; Costa (2014) encontró que solo 5 de las empresas estudiadas había puesto en práctica algún formato de flashmob, sumando un total de 18.

En la Región de Asia el conjunto de datos localizado por Wang, Akella y Bennett (2014), reveló que los flashmob en Asia habían aumentado de 5 en 2009 a 42 en 2011. Se identificaron 100 actividades en siete categorías (ver tabla 3). 
FLASHMOB COMO ESTRATEGIA DE MARKETING EN TABASCO, MÉXICO

Tabla 3

Actividades de flashmob en la Región Asiática

\begin{tabular}{lll}
\hline Categoría & Número flashmob & Mobber \\
\hline Personal & 41 & 7759 \\
Educacional & 17 & 6703 \\
Caridad & 11 & 1520 \\
Actuación artística & 10 & 1183 \\
Negocios & 6 & 685 \\
Política & 5 & 526 \\
Criminal & 1 & 70 \\
\hline
\end{tabular}

Fuente: Wang, Akella y Bennett (2014).

En América del Norte, los flashmob habían aumentado 3.6 veces de 10 en 2008 a 36 en 2011. Se localizaron 97 en diferentes categorías (ver tabla 4).

Tabla 4

Actividades de flashmob en América del Norte

\begin{tabular}{lll}
\hline Categoría & Número flashmob & Mobber \\
\hline Personal & 8 & $\mathrm{n} / \mathrm{d}$ \\
Educacional & 4 & $\mathrm{n} / \mathrm{d}$ \\
Caridad & 1 & $\mathrm{n} / \mathrm{d}$ \\
Actuación artística & 48 & $\mathrm{n} / \mathrm{d}$ \\
Negocios & 6 & $\mathrm{n} / \mathrm{d}$ \\
Política & 5 & $\mathrm{n} / \mathrm{d}$ \\
Criminal & 10 & $\mathrm{n} / \mathrm{d}$ \\
\hline
\end{tabular}

Fuente: Wang, Akella y Bennett (2014). 


\section{MATERIAL Y MÉTODO}

Se utilizó el método cualitativo con un enfoque fenomenológico sistémico, asumiendo que la fenomenología orienta a los científicos sociales a comprender los marcos codificadores e interpretativo de un fenómeno de interés (Martínez, 2006), como el uso de la estrategia de marketing innovadora del flashmob corporativo, en empresas mexicanas y tabasqueñas. El estudio se desarrolló con las técnicas de revisión literaria y análisis de contenido.

\section{RESULTADOS}

Replicando la metodología de investigación de Costa (2014), las autoras realizaron una búsqueda durante los meses de abril y mayo 2017, en diferentes canales de YouTube, libres y corporativos. Los hallazgos fueron que diversas empresas mexicanas y que operan en México están aplicando actividades de flashmob como medio de publicidad a través del espectáculo. En esta investigación se identificaron cinco categorías: Política, educacional, personal, actuación artística y de negocios. Para fines de este artículo, solo se consideró el análisis de esta última categoría. Se revisaron un total de 21 videos pertenecientes a seis empresas. El periodo de realización de actividades de flashmob en las organizaciones localizadas abarca de 2012 a 2016. Se encontraron un total de 20 actividades de flashmob en la categoría de negocios, las cuales tuvieron lugar en 8 ciudades del país (Ver tabla 5).

\section{Tabla 5}

Actividades de flashmob de negocios en México 2012-2016

\begin{tabular}{lcll}
\hline Empresa & Número flashmob & Lugar & Característica (temática) \\
\hline Palacio de & 3 & Monterrey, Nuevo León & $\begin{array}{l}\text { Años 80 (Noche Palacio Disco) } \\
\text { Ópera } \\
\text { Hierro }\end{array}$ \\
& 1 & Guadalajara, Jalisco & $\begin{array}{l}\text { Orquesta infantil } \\
\text { Tropical (Noches Palacio Tropical) } \\
\end{array}$ \\
& 1 & Puebla, Puebla & Orquesta \\
Subtotal & $\mathbf{6}$ & & \\
Liverpool & 1 & Monterrey, Nuevo León & Navidad \\
& 1 & Serdán, Puebla & Villancicos \\
Subtotal & 2 & & Navidad \\
Comercial & 2 & Ciudad de México & \\
Mexicana & & & \\
\hline
\end{tabular}




\begin{tabular}{llll}
\hline $\begin{array}{l}\text { Subtotal } \\
\text { Interjet }\end{array}$ & 2 & Atizapán, Hidalgo & Mes patrio \\
& 4 & Aeropuerto Ciudad de & Navidad \\
Subtotal & 1 & México & \\
Telcel & 3 & Guadalajara, Jalisco & Día de las madres \\
& & & Día del amor y la amistad \\
& 1 & Navidad & \\
& 4 & & Navidad \\
Subtotal & 1 & Cuernavaca, Morelos & Musical \\
Seat & 2 & Ciudad de México & Musical \\
Subtotal & 3 & & \\
Total & 20 & & \\
\hline
\end{tabular}

Fuente: Elaboración con datos de Bayardo (2012), Comer (2014); Cornejo (2013); Dos22 (2015), Eventos (2014); GDL (2014); GDL (2015); Interjet (2013); Morelos (2012); Muñoz (2013); Oficial (2016); Producciones (2013); Producciones (2014a), Producciones (2014b); Romero (2015); Sowubani (2011), Sorjuana1000 (2011a); Sorjuana1000 (2011b); Tuaudio (2016).

Con miras a conocer las potencialidades del uso del flashmob en Tabasco, se recurrió a la misma técnica de investigación que en las empresas mexicanas, se revisaron diversos canales de YouTube y solo se localizaron 3 videos que dan cuenta de esta actividad, cuyo lugar sede fue la ciudad de Villahermosa (ver tabla 6).

Tabla 6

Actividades de flashmob en Villahermosa, Tabasco, México

\begin{tabular}{|c|c|c|c|}
\hline Categoría & Año & Organización & Tema \\
\hline Caridad & 2012 & SPES & $\begin{array}{l}\text { Sensibilización social acerca de la } \\
\text { discapacidad física }\end{array}$ \\
\hline Actuación Artística & 2013 & Grupo de Danza & Diversas coreografías \\
\hline $\begin{array}{l}\text { Educacional } \\
\text { (turístico) }\end{array}$ & 2015 & $\begin{array}{l}\text { Gobierno del Estado (3er. } \\
\text { Festival del Queso) }\end{array}$ & $\begin{array}{l}\text { Danza folclórica con temas } \\
\text { culturales de la sede del Festival }\end{array}$ \\
\hline
\end{tabular}

Fuente: Elaboración con datos de ForoSPES (2012); Festival Queso (2015); Montejo (2013). 


\section{Potencialidades del flashmob en Tabasco, México}

Se aprecia que el uso del flashmob en Tabasco, México tiene potencialidades diversas en uso, particularmente los de tipo corporativa, artística educacional y de caridad; sin embargo, las nuevas tendencias evidencian la necesidad de estrategias innovadoras en el sector turístico, donde el flashmob cubriría los requerimientos de comunicaciones integradas del marketing, en un formato creativo que ayudara a posicionar los productos y recursos turísticos de la entidad.

\section{CONCLUSIONES}

El flashmob es una técnica única y original con uso en varias áreas como social, político, artístico, religioso, educación, personal y negocios; así considerada como una nueva estrategia de marketing que sirve para hacer publicidad de un cierto producto, servicio y hasta hacer conciencia de la propia marca.

En diversas partes del mundo esta estrategia ya ha sido aplicada y se obtenido resultados rentables. Europa, Asia y América del Norte son las regiones donde más presencia tiene. Desde 2014 cinco grandes empresas españolas utilizan el flashmob y la viralizan por medio de videos publicados en su canal de YouTube; en Asia en el año 2011 ya se llevaba 100 flashmobs en práctica pero en diferente categorías, para el mismo año en la región de América del norte, Estados Unidos tuvo la mayor frecuencia con un total de 97 eventos; y México sumó un total de 15 flashmob.

Se replicó la técnica de investigación de Costa (2014) y se analizaron empresas mexicanas o con presencia en México, que utilizaban esta técnica como estrategia. Se identificaron 21 videos en canales de YouTube correspondiente a seis grandes corporativos. Estas actividades tuvieron lugar en ocho ciudades del país.

Por lo tanto, se concluye que las actividades de esta estrategia de marketing son mayormente utilizadas en países desarrollados y en general por grandes corporativos. En el caso de Tabasco, solo se encontraron 3 videos que dan cuenta de actividades de flashmob en las categorías de caridad, actuación artística y educacional (turismo). En general, se puede asumir que esta técnica tiene un gran potencial para ser aplicada en esta región del país, especialmente en la categoría educacional y al turismo. 
FLASHMOB COMO ESTRATEGIA DE MARKETING EN TABASCO, MÉXICO

\section{REFERENCIAS BIBLIOGRÁFICAS}

Andrade Yejas, D. A. (Enero-Junio de 2016). Estrategias de marketing digital en la promoción de Marca Ciudad. Escuela de Administración de Negocios (80), 59-72.

Bayardo, E. (17 de Diciembre de 2012). Flashmob La Superior en el Palacio de Hierro, ESMDM. Recuperado el 2017, de https://www.youtube.com/watch?v=gcFfsMnZKjg

Catalá, M., \& Díaz, O. (2013). Publicidad 360. Zaragoza, España: Ediciones Universidad San Jorge.

Comer, L. (13 de Diciembre de 2014). Flashmob en La Comer. Recuperado el 2017, de https://www.youtube.com/watch?v=vXnejmCXhUw

Córdoba López, J. F. (Enero-Julio de 2009). Del marketing transaccional al marketing relacional. Entramado, 5(1), 6-17.

Cornejo, M. (2 de Septiembre de 2013). FlashMob Comercial Mexicana. Recuperado el 2017, de https://www.youtube.com/watch?v=qa81Qlau0bg

Costa Sánchez, C. (2014). Flash Mob Corporativo. Estudio de su empleo por las empresas españolas con mejor reputación. Journal, 8(4), 71-90.

Dos22, R. P. (28 de Diciembre de 2015). Liverpool Galerías Serdán: Flash Mob Navideño. Recuperado el 2017, de https://www.youtube.com/watch?v=DGOPBZDJYsI\&t=163s

Eventos, S. (6 de Diciembre de 2014). FlashMob Palacio de Hierro Monterrey Noches Palacio Disco. Recuperado el 2017, de https://www.youtube.com/watch?v=XWz8z6QwQul

Estrella Ramón, A. \& Segovia López, C. (2016). Comunicación integrada de marketing. Madrid: ESIC Editorial. Estrella Ramón, A. \& Segovia López, C. (2016). Comunicación integrada de marketing. Madrid: ESIC Editorial.

Festivalqueso. (7 de Agosto de 2015). FLASH MOB 3ER FESTIVAL DEL QUESO ARTESANAL. Recuperado el 2017, de https://www.youtube.com/watch?v=cr_B9tk6DOE\&t=1s

ForoSPES (5 de Marzo de 2012). SPES Flashmob. Recuperado el 2017, de https://www.youtube.com/watch?v=r7v3gy9i5Ts\&t=4s

Fraile Prieto, T. (2012). Músicas para persuadir. Apropiaciones musicales e hibridaciones genéricas en la publicidad audiovisual. Comunicación, 324-337.

Gamboa Pastrana, S. (2015). Repositorio Educativo Digital. Recuperado el 26 de Febrero de 2017, de EL FLASHMOB: De la expresión ártistica a la estrategia: http://red.uao.edu.co:8080/handle/10614/8261 
FLASHMOB COMO ESTRATEGIA DE MARKETING EN TABASCO, MÉXICO

GDL, F. (25 de Diciembre de 2014). Flashmob en Plaza del Sol Diciembre 2014. Recuperado el 2017, de https://www.youtube.com/watch?v=SW98I_b8CQk

GDL, F. (11 de Enero de 2015). Flashmob Morelia Mich. 4 Enero 2015. Recuperado el 2017, de https://www.youtube.com/watch?v=CTc6YNiygRM

Jiménez Diez, O. (s.f.). Marketing de contenido, una estrategia de lealtad. Revista PYME AH, 20-23. Jiménez Diez, O. (s.f.). Marketing de contenido, una estrategia de lealtad. Revista PYME AH, 20-23.

Interjet. (24 de Diciembre de 2013). FlashMob Interjet ¡Feliz Navidad y Prospero Año Nuevo! Recuperado el 2017, de https://www.youtube.com/watch?v=OWLL-aSob4U

Jiménez Diez, O. (s.f.). Marketing de contenido. PYME, 20-23.

López García, S. (2015). Arte callejero vs. Street Marketing. Recuperado el 01 de Marzo de 2017, de https://uvadoc.uva.es/handle/10324/14089

Martínez Miguelez, M. (2006). Ciencia y Arte en la Metodología Cualitativa (Sda. Ed.). México: Trillas

Montejo, E. (24 de Marzo de 2013). Plaza altabrisa danza 3 studio. Recuperado el 2017, de https://www.youtube.com/watch?v=JD1kA2rdKao\&t=168s

Morelos, F. (17 de Diciembre de 2012). FLASHMOB "SEAT" 2012. Recuperado el 2017, de https://www.youtube.com/watch?v=m7_-Jv9fY18

Muñoz, C. (14 de Agosto de 2013). Behance. Recuperado el 2017, de Flashmob Palacio de Hierro Guadalajara: https://www.behance.net/gallery/10393543/Flashmob-Palacio-deHierro-Guadalajara.

Oficial, C. M. (17 de Diciembre de 2016). Flashmob Navideño. Recuperado el 2017, de https://www.youtube.com/watch?v=t4XuWcPbShA\&index=13\&list=PL_K2HWI3TuCB0CIlrv aFomHOzFRP_z7ws

Patfr73. (8 de Mayo de 2013). Rumbo al Urban Test Drive SEAT D.F. Six flags. Recuperado el 2017, de https://www.youtube.com/watch?v=JP7ptfO5-ZU

Pérez Serrano, M. J., \& Fanjul Peyró, C. (2013). El valor de la creatividad en la comunicación publicitaria audiovisual de calidad. Sociedad consumo y marca. Razón y Palabra (85), 18.

Periodismouma.es (2015). Tipos de flashmob. Recuperado el 17 de junio de 2017 de http://www.periodismo.uma.es/perint1112/FLASHMOB/tipos.html

Producciones, E. (17 de Mayo de 2013). FLASHMOB SEAT 2013 México. Recuperado el 2017, de https://www.youtube.com/watch?v=L2uBXeyx6Ak 
FLASHMOB COMO ESTRATEGIA DE MARKETING EN TABASCO, MÉXICO

Producciones, M. (9 de Julio de 2014a). FLASH MOB TELCEL PLAZA DEL SOL. Recuperado el 2017, de https://www.youtube.com/watch?v=X_ZYld-W3bE

Producciones, M. (27 de Febrero de 2014b). FlashMob Telcel Día del Amor y la Amistad. Recuperado el 2017, de https://www.youtube.com/watch?v=vkOLMqv_nBo

Romero, L. F. (11 de Diciembre de 2015). Flashmob Navideño en Liverpool Galerias Monterrey. Recuperado el 2017, de https://www.youtube.com/watch?v=PIPa03s4Qaw

Sorjuana1000. (22 de Noviembre de 2011a). Baile en Palacio de Hierro Monterrey. Recuperado el 2017, de https://www.youtube.com/watch?v=3FqT7ots9j0

Sorjuana1000. (13 de Junio de 2011b). Palacio de Hierro Monterrey Opera 1.flv. Recuperado el 2017, de https://www.youtube.com/watch?v=5zhZAnxEMhs

Sowubani. (11 de Septiembre de 2011). flashmob Comercial Mexicana Atizapan. Recuperado el 2017, de https://www.youtube.com/watch?v=cCXY83zkZ_k

Tuaudio, T. (30 de Marzo de 2016). Flash Move Palacio De Hierro Puebla (Noches Palacio). Recuperado el 2017, de https://www.youtube.com/watch?v=qIABx8DIDoM

Viteri Vallejo, J. A. (2011). DSpace. (Q. EPN, Ed.) Recuperado el 21 de Marzo de 2017, de Estrategias de Inbound Marketing y Propuesta de Implementación para el Portal de Todocomercioexterior: http://bibdigital.epn.edu.ec/bitstream/15000/7711/1/CD-4373.pdf

Wang, C.-P., Akella, D., \& Bennett, C. (May de 2014). Flash Mobs in the 21st Century: Mobile Technology Shapes Human Collective Behavior. International Journal of Business, Humanities and Technology, 4(3), 24-32. 